\title{
DEVELOPMENT OF SOFTWARE AND HARDWARE SYSTEMS VERBAL INTELLIGENT ROBOT
}

\author{
G.M.AYAZBAEV ${ }^{1}$, A.V.KIM ${ }^{2}$ \& M.KUNELBAYEV ${ }^{3}$ \\ ${ }^{1}$ Kazakh National University. Al-Farabi Kazakh National University, Almaty, Republic of Kazakhstan \\ ${ }^{2}$ Institute of Information and Computer Technology (ICE), Almaty The Republic of Kazakhstan
}

\begin{abstract}
This article discusses the main approaches to create intelligent robots. Shows the architecture of voice command systems in complex verbal robot. Results of development of software and hardware complex verbal robot. The results are shown in simulations using robots with three-dimensional modeling software packages.

KEYWORDS: Robotics, Humanoid, Collaborative Robots, Electronics, Programming, Intelligent Systems
\end{abstract}

Received: Jun 01, 2020; Accepted: Jun 20, 2020; Published: Jul 02, 2020; Paper Id.: IJMPERDJUN2020169

\section{INTRODUCTION}

Modern robotics far stepped forward. Robots have become an integral part of our daily life. Today they can be not only physical, but also intelligent human helpers. More and more places and professions, where their presence has greatly improved and the use of many production, manufacturing processes, making them more technologically advanced and safe. New trend for modern robots is their use in the areas of: education, medicine, entertainment, advertising, commercial activities in space, in the military field, as well as during emergencies.

In everyday life, are increasingly found the anthropomorphic (humanoid) robots, capable of interactive communication verbal and nonverbal nature with people. Many of them have artificial intelligence that can understand, answer questions, talk and even make small talk. However, as a rule, they are all focused on the international language of communication - English, at least in the Japanese, Chinese, Russian and other languages.

To date, it has not yet developed the software for robots that can understand and speak the Kazakh language. Also little known study on the practical application of verbal (voice) command and control and manipulation of mobile robotic systems. However, developments in this direction are active in Kazakhstan in ICE [1,2].

The purpose of this research is to develop a universal hardware and software (APC) intelligent robot:

\section{To Control Robots of Different Types:}

- $\quad$ Androidnye (service);

- $\quad$ manipulation (Collaborative);

- $\quad$ mobile (terrestrial wheel). 
For Verbal Control Robotic Systems Separately and Simultaneously able to Understand Voice Commands in One of Three Languages:

- $\quad$ - English;

- Russian;

- - Kazakh;

To Perform Controlled Movements of the Robotic Systems Control Voice Commands in the Verbal Mode in One Of Three Languages:

- English;

- Russian;

- Kazakh;

Recognition of human speech - it is a difficult technical problem, in which the sound (acoustic) signals are analyzed and structured into a hierarchy of words, phrases and sentences. The main problems of speech recognition are: acoustic and temporal variability that at different times the same voice audio files have different characteristics in amplitude, frequency, tone, duration, etc. [3,4].

There are basic methods of speech recognition [5]:

- $\quad$ Hidden Markov Model;

- Dynamic time warp;

- Artificial Neural Networks.

Artificial neural networks (ANN) - mathematical models and their software or hardware implementation, built on the principle of the organization and functioning of biological neural networks. These models have emerged in the study of the processes occurring in the human brain, and when you try to simulate these processes. The first such attempt was neural networks McCulloch and Pitts. Later, after the development of learning algorithms created by the model began to be used in many practical problems.

INS system are connected and interacting simple processors (artificial neurons). Such processors are usually quite simple, especially in comparison with the CPUs used in personal computers. Each processor of such a network has to deal only with signals that it regularly receives, and signals that he periodically sent to other processors. At the same time, being connected by a fairly large network with controlled interaction of such locally simple processors together are able to perform quite complex tasks.

In terms of machine learning, neural network is a special case of pattern recognition methods, discriminant analysis, clustering methods, etc. From a mathematical point of view, the neural network training. This multiparameter problem of nonlinear optimization.

From the point of view of cybernetics, a neural network is used in problems of adaptive control algorithms and as a basis for robotics. 
In terms of computer technology and programming, neural network - a way to efficiently parallelize tasks.

From the viewpoint of artificial intelligence, ANN is philosophical basis connectionism flow and main direction in the structural approach to explore the possibility of constructing (simulation) of natural intelligence using computer algorithms.

Neural networks are not programmed in the usual sense of the word, they are trained. The possibility of training one of the major advantages of neural networks over conventional algorithms. Technical training is to find the coefficients of the connections between neurons. During training, the neural network is able to identify complex dependencies between the input and output data, and perform synthesis. This means that in case of successful learning network will be able to return the correct result on the basis of data that were not available in the training set, as well as incomplete and / or "noisy", partly distorted data. Humanoid robots have a human-like design and are able to imitate human movements [7]. Humanoid robots with double manipulators and dexterous hands shows great importance in all maintenance applications, as well as in medical. Many humanoid robots have been built over the past few years, such as ASIMO [8,9,10], HPR [11], WABIANRV [12], NAO [13,14,15] and the last "pepper" robot [ 16,17]. Some humanoid robots are made of metal, steel or aluminum, which are too heavy like a CALUMA robot [18]. To interact with a person. robots need a light structure in order to adapt their configuration conveniently and be energy-efficient [19]. Currently, one of the most rapidly developed technologies in recent years is $3 \mathrm{D}$ printing [20]. Thanks to 3D printing technology, it is possible to quickly develop an idea from a virtual to a real prototype. Some humanoid robots like Flobi [21] and iCub [22] take advantage of 3D printing, but they are partial 3D printing.

\section{METHODS OF CALCULATION}

Range of human speech is $20 \mathrm{~Hz}-20 \mathrm{kHz}[3,4,5]$. Original speech signal is represented in discrete form as:

$$
x[n], 0 \leq n<N
$$

Applying the Fourier transformation to it:

$$
X_{a}[k]=\sum_{n=0}^{N-1} x[n] e^{\frac{-2 \pi i}{N} k n}, 0 \leq k<N
$$

Calculate the frequency value in the mel-scale:

$$
B^{-1}(b)=700(\exp (b / 1125)-1)
$$

We compute the energy of the signal:

$$
S[m]=\ln \left(\sum_{k=0}^{N-1}\left|X_{a}[k]\right|^{2} H_{m}[k]\right), 0 \leq m<M
$$

Apply the DCT and obtain the feature set of the signal coefficients MFCC:

$$
c[n]=\sum_{m=0}^{M-1} S[m] \cos (\pi n(m+1 / 2) / M), 0 \leq n<M
$$

The result of analysis is a signal sequence of 10 miles-second speech frames containing information about these portions coefficients MFCC. To improve the quality of recognition in the frames can be added information on the first or second derivative values of coefficients to describe the dynamics of speech change $[3,4,5]$.

Acoustic units - phonemes, allophones, diphones, Trifonov [3].

\section{Acoustic Processing Steps}


- Segmentation,

- Feature extraction,

- Modeling of acoustic units,

Mel (from the Greek $\mu \varepsilon \lambda \mathrm{o} \varsigma$ - sound) - psychophysical unit of pitch, a quantitative assessment of the sound adjustment is based on the statistical treatment of a large number of data on the subjective perception of the height of the sound tones. Research results show that the pitch is mainly related to the oscillation frequency, but depends also on the tone volume level and tone [2,3].

In speech recognition, use a combination of the following models:

- Acoustic model which contains information about the audio parameters (attributes) of each Senonian; are both context-dependent and context-free. Denote the set of features both $\mathrm{O}$, then $\mathrm{W}$ for word probability of finding a set of $\mathrm{O}-\mathrm{P}(\mathrm{O} \mid \mathrm{W})[5,6]$.

- The phonetic dictionary containing a mapping from words into phonemes. It may be noted that the representation in the form of a dictionary is not required: it may be a certain function, the resulting machine learning algorithms. We denote as the pronunciation of the word W Q (W) [5,6].

- The language model, which limits the search words. It defines what the word may follow the data and with what probability, thus cutting off the impossible alternatives. The most commonly used n-gram Language Model. Denote the probability of finding a word $\mathrm{W}$ as the $\mathrm{P}(\mathrm{W})[5]$.

If we designate the original audio signal for $\mathrm{X}$, and the set of all words of $\mathrm{W}$, the speech recognition problem is formalized as follows [5]

$$
W^{*}=\underset{W \in \mathrm{W}}{\operatorname{argmax}} P(W \mid X)=\underset{W \in \mathrm{W}}{\operatorname{argmax}} P(O \mid Q(W)) P(W)
$$

In this study to determine the effectiveness of speech recognition systems used metric Word Error Rate (WER), based on the calculation of insertions, deletions and substitutions of words [3]

$W E R=\frac{S+D+I}{N}(7)$

where

$S$ - the number of substituted words

$D$ - the number of deleted words

$I$ - the number of inserted words

That is, the higher the WER, the less qualitative detection occurred. Usually expressed as a percentage.

There are several known speech recognition systems with open source: CMU Sphinx, HTK, Kaldi, Julius [3]. 


\section{CMU SPHINX SYSTEM}

At the moment, the actual version is sphinx4-5prealpha. Sphinx-4 is a modular framework. The modular structure allows to vary the parameters of the system based on the requirements of a particular task. Highlighted 3 main modules: Front End, Decoder and Linguist [1].

Front End converts the input data into a vector of parameters. Linguist based on the selected language, the acoustic models and vocabulary building Search Graph. Finally, Decoder'a submodule - Search Manager - uses a parameter vector and constructed graph for decoding and produces the result [1].

Front End combines several strings of modules communicating data processors, each capable of producing different parameters resulting calculations. Having multiple chains allows both to perform calculations for various types of parameters and to accept multiple input signals simultaneously [1].

Linguist. As mentioned above, the construction Search Graph'a in Linguist takes place on the basis of data about the language derived from language and acoustic models. Each of them is a HMM for elementary acoustic units used in a particular system. The dictionary associates words of a language model and an acoustic model combinations of elements [1].

The language model describes the structure of language on the level of words. Graph grammattiki and the ngrammnye model: The following two types of models are usually used. Graph grammmatiki represent a directed graph wherein vertices are word, and each edge corresponds to the weight, which is likely to move to the next word. In the case of n-grammnoy model has a set of probabilities to meet the word, if we know the previous n-1 words. In this paper we used trigram language model for US English. [6]

The dictionary contains pronunciations for all words in a given language model. Pronunciation of the word is broken into sets some basic blocks. For example, abandon $\leftrightarrow$ AH B AE ND AH N [6].

Acoustic model displays elements of speech (in this case - triphones) on the HMM. Naturally, the display can receive information about the context and the position in the word. The left and right contexts are discussed above, and the position information indicates whether Trifon is the beginning, middle or end of a word. Or is itself a word [6].

The Linguist breaks up each word in the context-sensitive elementary blocks (according to the dictionary), which then builds a lot of acoustic model HMM graphs. The vertices of these graphs - phonemes or triphones and ribs have a weight - the probability of transition between phonemes). And Search Graph [6] is based on the resulting graph and language moedli.

Search Graph - directed graph in which each vertex represents producing (emitting) or unproductive (non-emitting) state. Generating states correspond viewed sound features, and non-producing states are a high-level language constructs such as words or phonemes, which relate to the calculation of characteristics indirectly. Arcs of the graph represent transitions between states, each arc corresponds to a certain probability of passage through it [6].

Decoder. The main function of this module - for calculated in Front End features and built in Linguist'e Search Graph'u get a lot of hypotheses. Decoder sending submodule called Search Manager request to recognize a plurality of frames with the above data. At each stage Search Manager work builds all paths that reach a final non-productive state. Search Manager transmission algorithm uses tokens. For Search Manager algorithm used may, but need not, contain a plurality of active tokens (Active List). To simplify the calculations submodule Pruner held reduction of the set of tokens. Submodule Scorer on request computes estimates of the distribution of density of states for the data at given times [6]. 


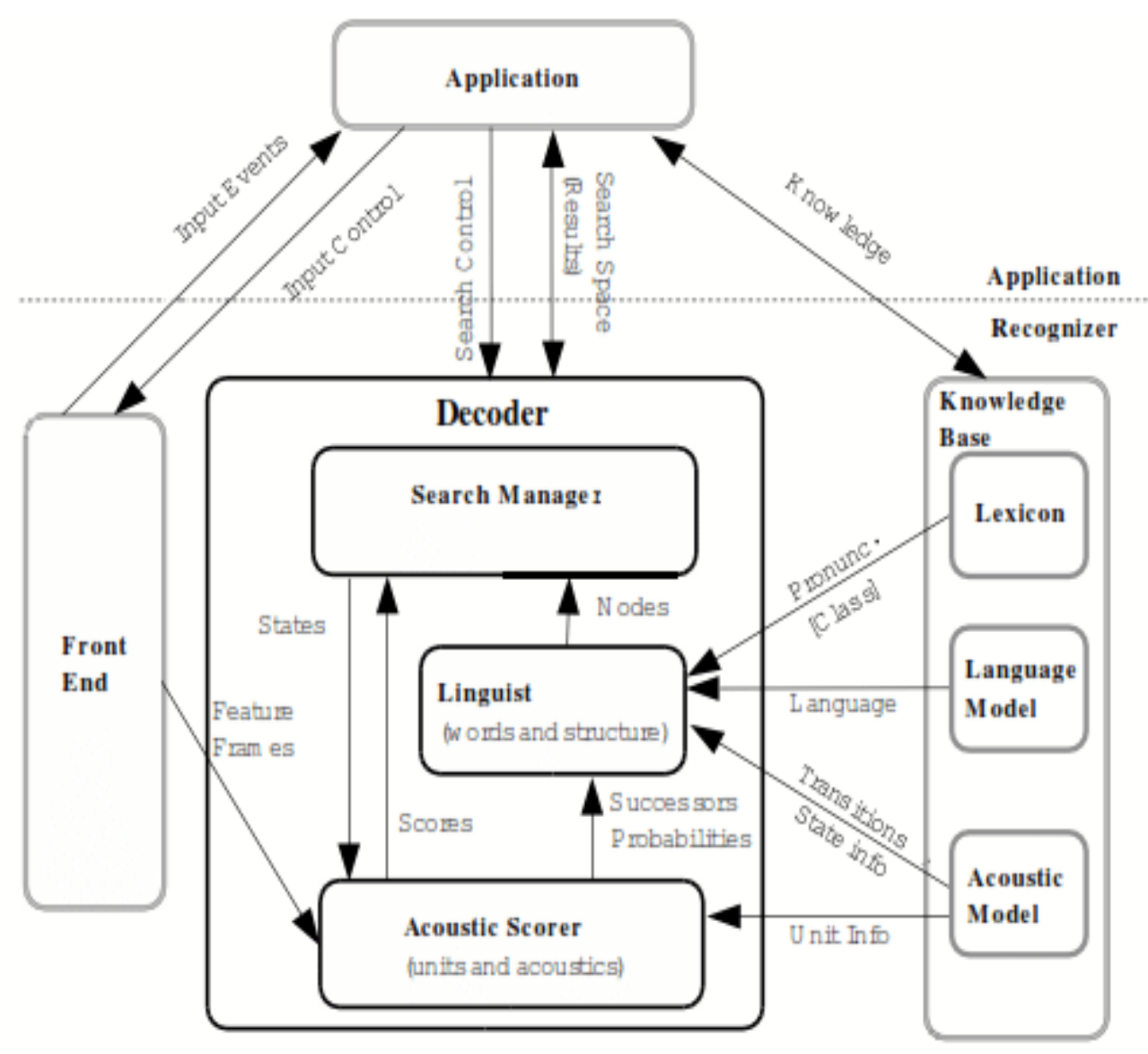

Figure 1: Architecture CMU Sphinx.

\section{EXPERIMENTS}

Data for the analysis were provided by the Laboratory "Computer Engineering intelligent systems." The data set consists of 1480 recordings of 20 speakers with 74-75 records. Each audio recording is made up of the phrases in the Kazakh language the average duration of 6 seconds. For the identification of the speaker, we had collected the following data: name, sex, place of birth, year of birth [4].

All audio materials have the same characteristics:

- File extension: wav;

- digital conversion method: PCM;

- $\quad$ sampling frequency: $44.1 \mathrm{kHz}$;

- capacity: 16 bits;

- the number of audio channels, one (mono).

Sound and recording one speaker took an average of 40-50 minutes, including the time needed for the preparation of dynamics, equipment and doublings, which corresponds to 74-75 receive a file for a total of 7-8 minutes for each speaker. [4] 
To conduct research and test the performance of the developed algorithms and software was created by a software simulator using three-dimensional modeling library Open LG (Figure 3-7).

CMU Sphinx implemented in C / C ++ language [1]. Intellectually the verbal complex was developed in Python with a communication module and a graphic simulator CMUSphinx execute commands Py Open GL. Robot models for the bases of three-dimensional simulation were chosen android and mobile robot models. Graphical results of the study are shown in Figures 3-6.

At the heart techniques work equipment is medium combining acoustic phoneme three languages (Kazakh, Russian, English). Fundamentally technique work does not differ between themselves That for English, Russian and Kazakh languages [1] [3]. The aim of the study was to test the methodology and quality of voice of speech recognition in three languages. The results obtained showed WER fluctuation in the range of $\sim 61-65 \%$, which is quite not a bad result for the first work on the language integration.

The robot control system module realized blocks recognition software voice commands to: Kazakh, Russian and English languages.

Block experiment control circuit shown in Figure 2.

Key instruction words in the database control blocks of data shown in Table 1.

Control block commands results are shown in Figure 3.

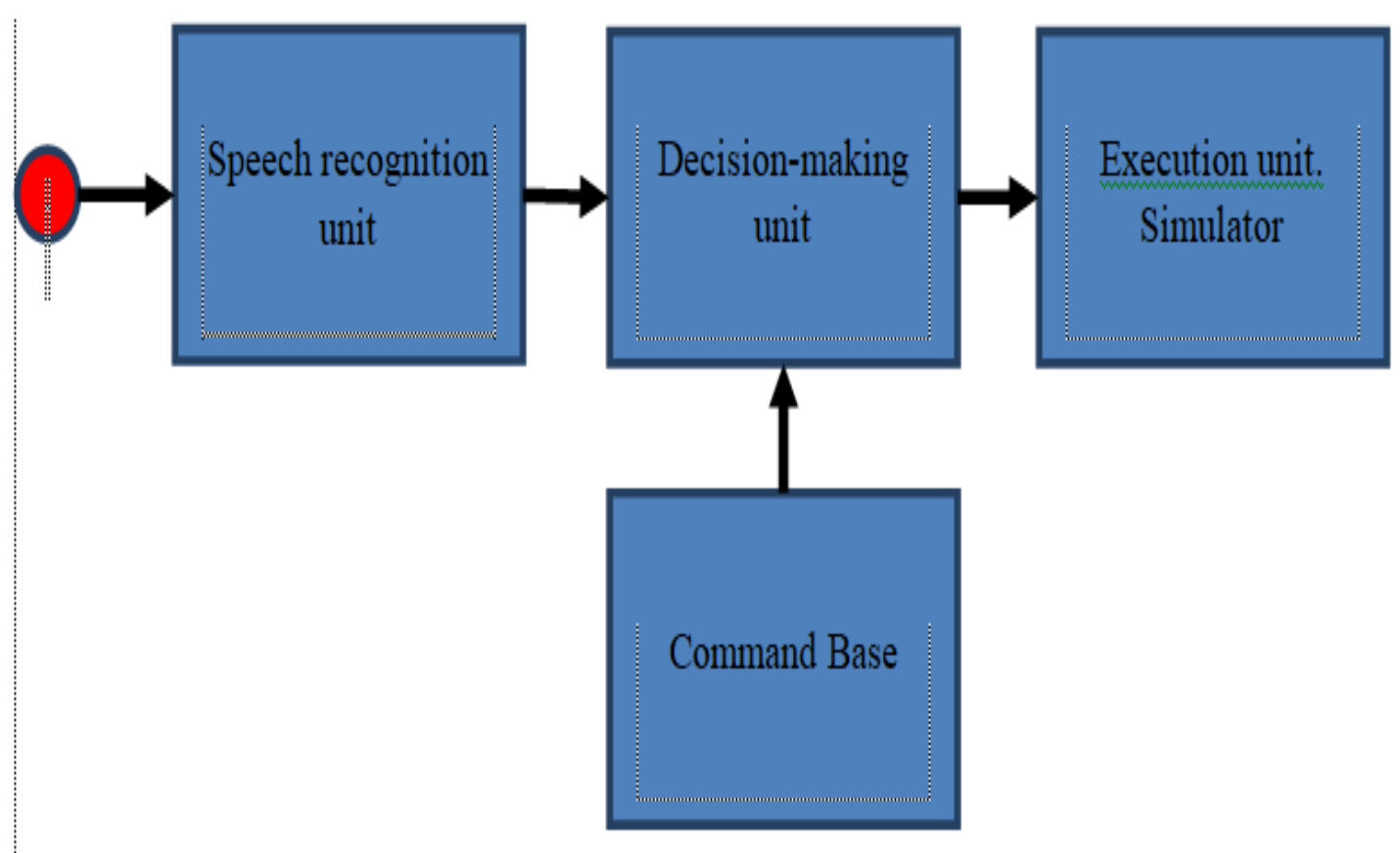

Figure 2: Block Diagram of the Control Experiment.

Table 1: Main Robot Control Commands

\begin{tabular}{|c|l|l|l|}
\hline S. No & Kazakh & \multicolumn{1}{|c|}{ Russian } & English \\
\hline 1 & Алға & Вперед & go \\
\hline 2 & Артқа & Назад & back \\
\hline 3 & Оңға & Направо & right \\
\hline 4 & Солға & Налево & left \\
\hline
\end{tabular}




\begin{tabular}{|l|l|l|l|}
\hline 5 & Тоқта & Стоять & stop \\
\hline 6 & Көтеру & поднять & catch up \\
\hline 7 & Төмендету & опустить & let go \\
\hline 8 & Алу & схватить & catch \\
\hline 9 & Қою & положить & put down \\
\hline
\end{tabular}

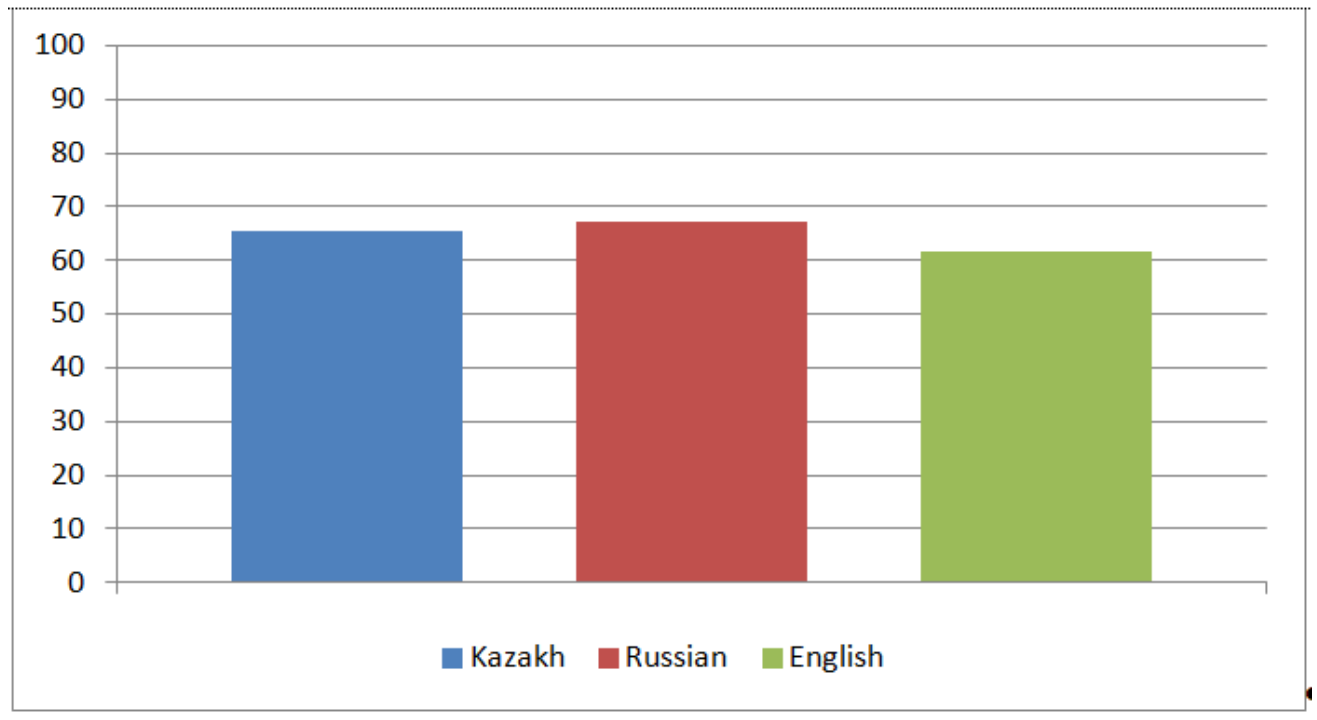

Figure 3: Results of the Robot Control Commands.

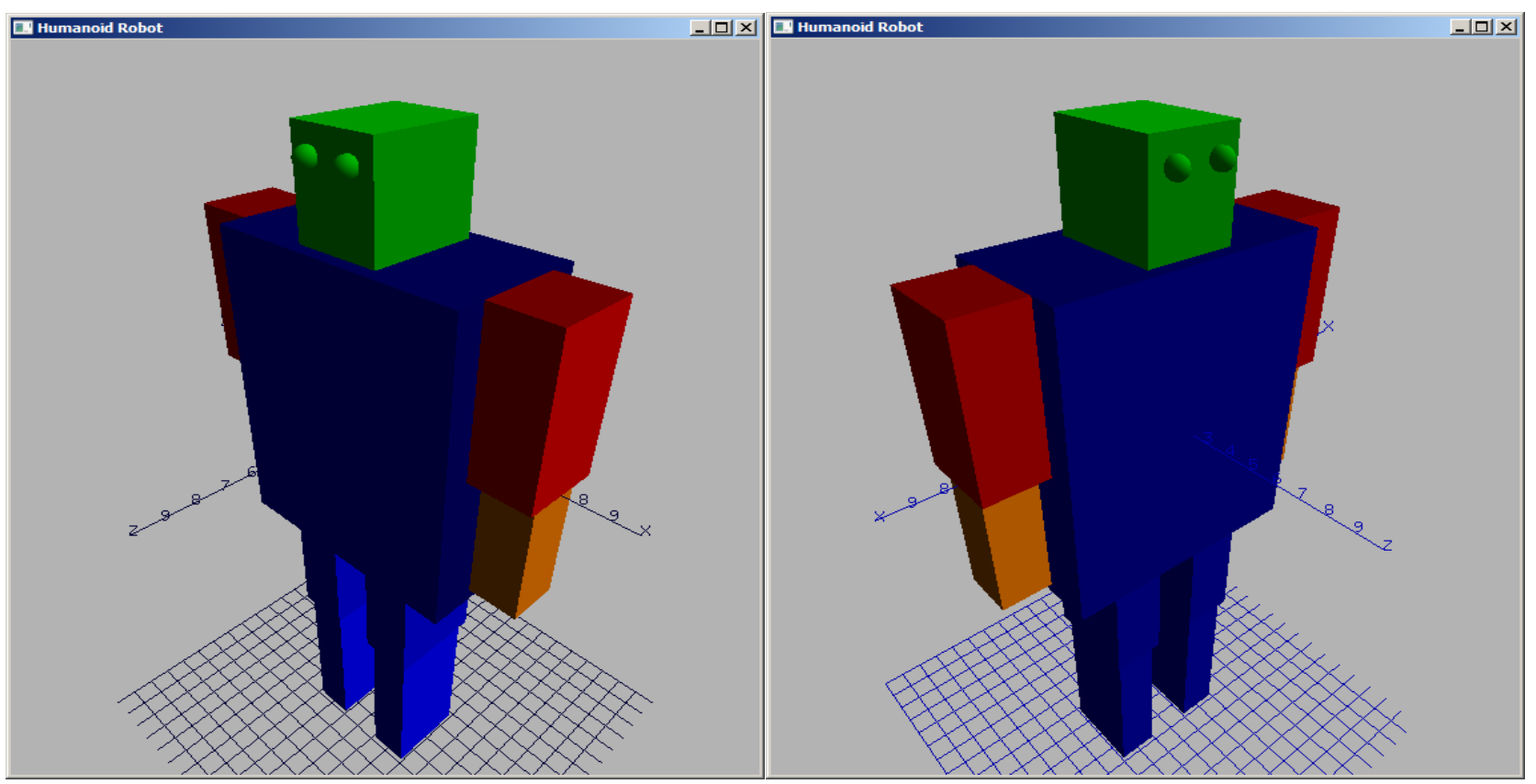

Figure 4: Simulation Android Robot Teams, "Left", "Right". 

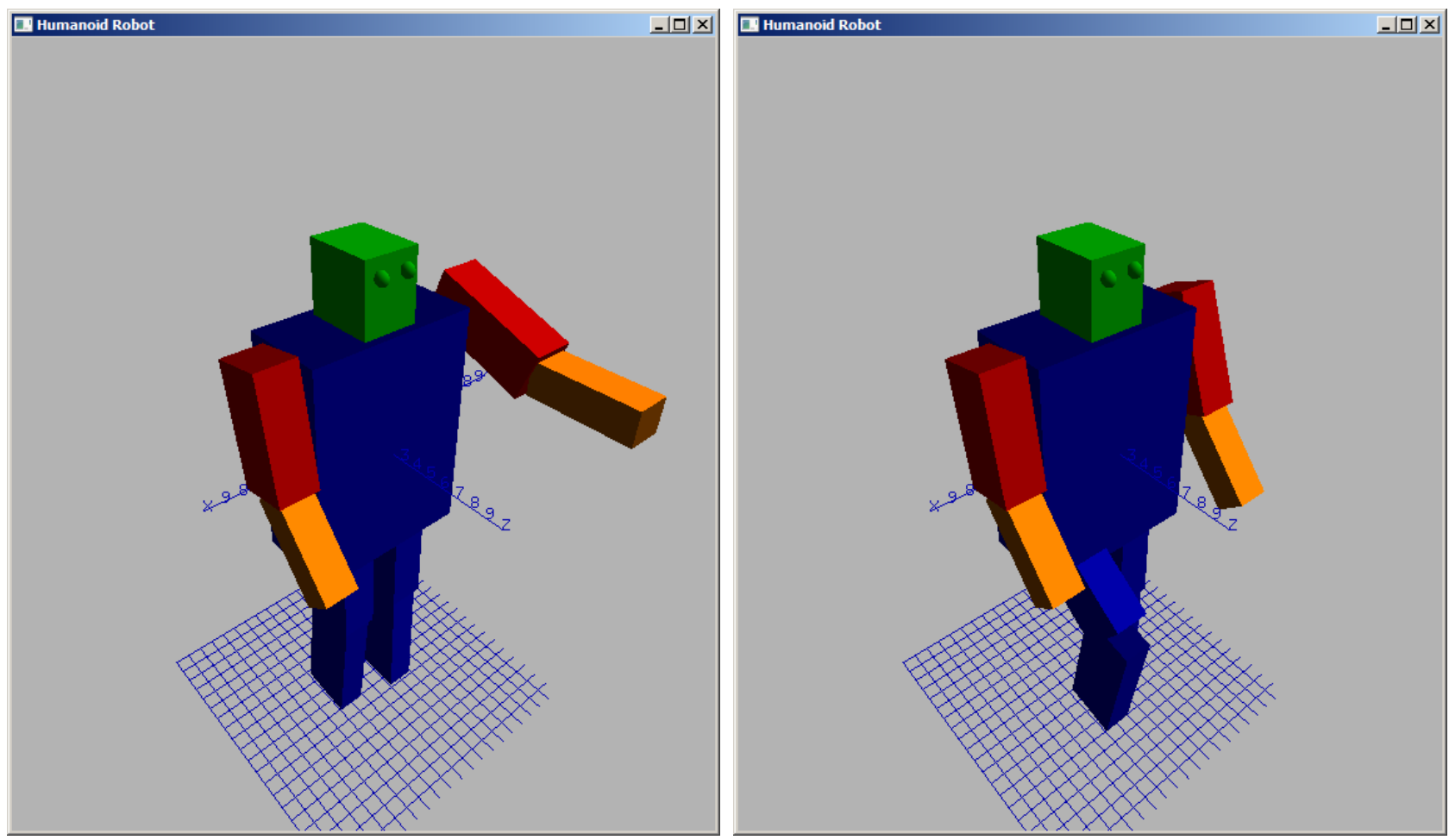

Figure 5: Simulation Android Robot Commands, "Forward", "Back".
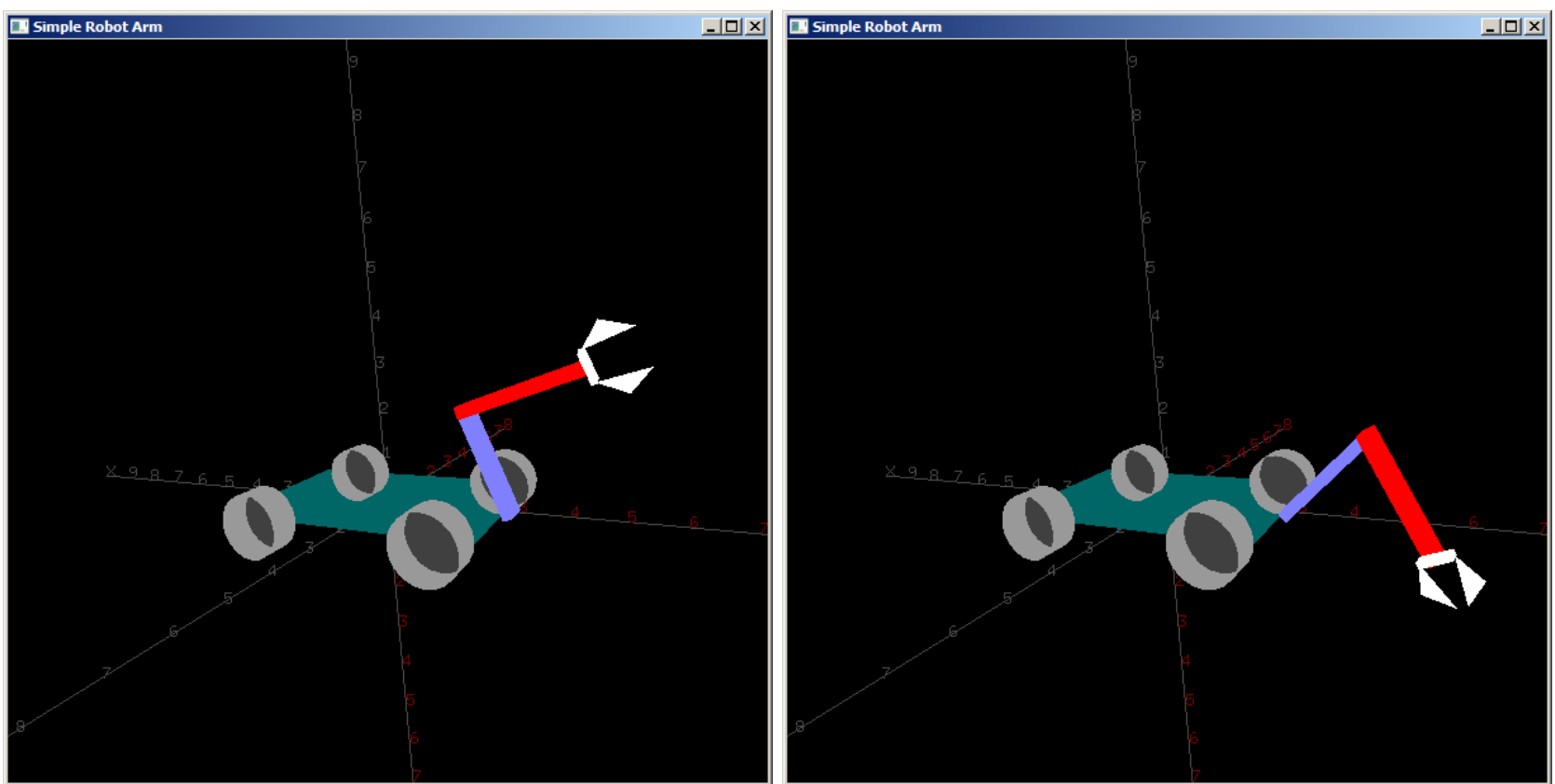

Figure 6: Simulation Manipulyatsinnogo Mobile Robot for the teams, "Lower", "Capture". 

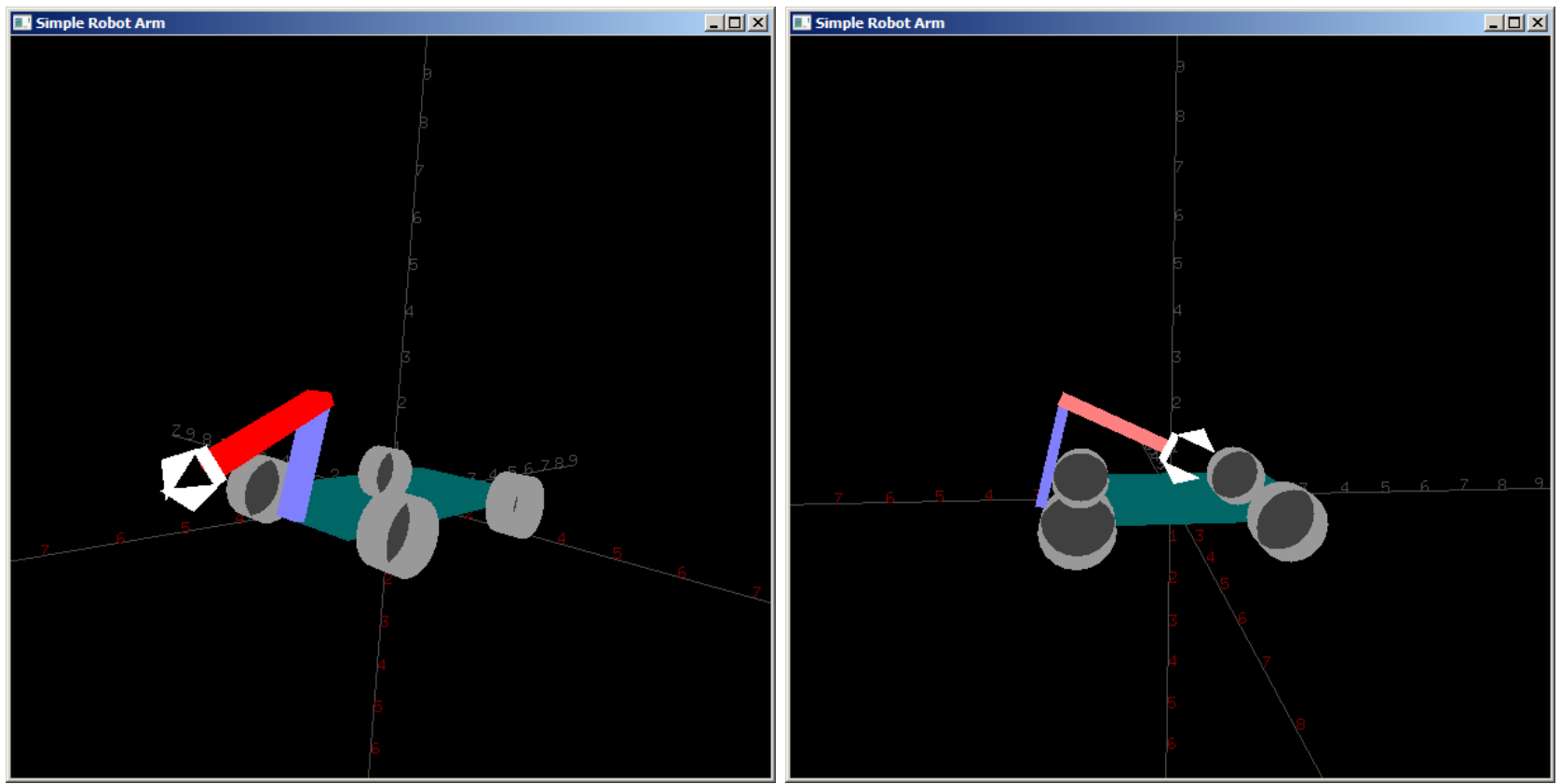

Figure 7: Simulation of the Manipulation of the Mobile Robot for the Teams, "Raise", "Put".

\section{CONCLUSIONS}

This article presents the results of studies conducted at the Institute of Information and Computer Technology (IIFT), the Ministry of Education and Science of the Republic of Kazakhstan (Almaty) on the development of hardware and software to build movements verbal anthropomorphic robots. On the basis of the three-dimensional simulation designed to study android, manipulator and mobile types of robots, we present the results of computer simulation of voice command and control in the Kazakh, Russian and English languages.

In addition, in the future it is planned to carry out further research in this direction, for the technical implementation of the universal software and hardware complex for the verbal command and control various types of robots: manipulation, mobile, anthropomorphic.

Particularly relevant and interesting for many developers and experts in the field of robotics, robotics in recent years is a collaborative mode (group) of business process management in a variety of service industries and the integration of technology in the production technology collaboration.

\section{REFERENCES}

1. CMU Sphinx Project by Carnegie Mellon University.http://cmusphinx.sourceforge.net/

2. Orken Mamyrbayev, Mussa Turdalyuly, Nurbapa Mekebayev, Keylan Alimhan, Aizat Kydyrbekova, Tolganay Turdalykyzy. Automatic Recognition of Kazakh Speech Using Deep Neural Networks // Asian Conference on Intelligent Information and Database Systems, pp, 465- 474, 2019

3. Orken Mamyrbayev, Mussa Turdalyuly, Nurbapa Mekebayev, Kuralay Mukhsina, Alimukhan Keylan, Bagher BabaAli, Gulnaz Nabieva, Aigerim Duisenbayeva, Bekturgan Akhmetov. Continuous Speech Recognition of Kazakh Language // ITM Web of Conferences №24, 2019 
4. Orken Mamyrbayev, Nurbapa Mekebayev, Mussa Turdalyuly, Nurzhamal Oshanova, Tolga Ihsan Medeni, Aigerim Yessentay. Voice Identification Using Classification Algorithms // Intelligent System and Computing, IntechOpen, pp.1-10, 2019

5. Xuedong Huang, Alex Acero, Hsiao-Wuen Hon, Spoken Language Processing: A Guide to Theory, Algorithm, and System Development, Prentice Hall, pp. 567-675, 2001

6. Mikhailov Dmitrii. Analysis of CMUSphinx system performance // Saint-Petersburg, pp.10-20, 2016

7. N. Rodriguez, G. Carbone, and M. Ceccarelli, “Antropomorphic design and operation of a new low-cost humanoid robot," in Biomedical Robotics and Biomechatronics, 2006. BioRob 2006. The First IEEE/RAS-EMBS International Conference on, Feb 2006, pp. 933-938.

8. K. Hirai, M. Hirose, Y. Haikawa, and T. Takenaka, "The development of honda humanoid robot," in Robotics and Automation, 1998. Proceedings. 1998 IEEE International Conference on, vol. 2, May 1998, pp. 1321-1326 vol.2.

9. Y. Sakagami, R. Watanabe, C. Aoyama, S. Matsunaga, N. Higaki, and K. Fujimura, "The intelligent asimo: system overview and integration," in Intelligent Robots and Systems, 2002. IEEE/RSJ International Conference on, vol. 3, 2002, pp. 2478-2483 vol.3.

10. S. Okita, V. Ng-Thow-Hing, and R. Sarvadevabhatla, "Learning together: Asimo developing an interactive learning partnership with children, " in Robot and Human Interactive Communication, 2009. ROMAN 2009. The 18th IEEE International Symposium on, Sept 2009, pp. 1125-1130.

11. K. Kaneko, F. Kanehiro, S. Kajita, K. Yokoyama, K. Akachi, T. Kawasaki, S. Ota, and T. Isozumi, "Design of prototype humanoid robotics platform for hrp," in Intelligent Robots and Systems, 2002. IEEE/RSJ International Conference on, vol. 3, 2002, pp. 2431-2436 vol.3.

12. G. Carbone, H. ok Lim, A. Takanishi, and M. Ceccarelli, "Numerical and experimental estimation of stiffness performances for the humanoid robot wabian-rv," in Advanced Intelligent Mechatronics, 2003. AIM 2003. Proceedings. 2003 IEEE/ASME International Conference on, vol. 2, July 2003, pp. 962-967 vol.2.

13. P. Ghassemi, M. Masouleh, and A. Kalhor, "Push recovery for nao humanoid robot," in Robotics and Mechatronics (ICRoM), 2014 Second RSI/ISM International Conference on, Oct 2014, pp. 035-040.

14. S. Nugroho, A. Prihatmanto, and A. Rohman, "Design and implementation of kinematics model and trajectory planning for nao humanoid robot in a tic-tac-toe board game," in System Engineering and Technology (ICSET), 2014 IEEE 4th International Conference on, vol. 4, Nov 2014, pp. 1-7.

15. D. Lopez Recio, L. Marquez Segura, E. Marquez Segura, and A. Waern, “The nao models for the elderly," in Human-Robot Interaction (HRI), 2013 8th ACM/IEEE International Conference on, March 2013, pp. 187-188.

16. J. Lafaye, C. Collette, and P.-B. Wieber, "Model predictive control for tilt recovery of an omnidirectional wheeled humanoid robot," in Robotics and Automation (ICRA), 2015 IEEE International Conference on, May 2015, pp. 5134-5139.

17. J. Lafaye, D. Gouaillier, and P.-B. Wieber, "Linear model predictive control of the locomotion of pepper, a humanoid robot with omnidirectional wheels, " in Humanoid Robots (Humanoids), 2014 14th IEEERAS International Conference on, Nov 2014, pp. 336-341.

18. N. Eduardo, C. Giuseppe, and C. Marco, “Capaman2bis as trunk module in caluma (cassino low-cost humanoid robot)," in Robotics, Automation and Mechatronics, 2006 IEEE Conference on, Dec 2006, pp. 1-6.

19. D. Ye, S. Sun, J. Chen, and M. Luo, "The lightweight design of the humanoid robot frameworks based on evolutionary structural optimization, " in Robotics and Biomimetics (ROBIO), 2014 IEEE International Conference on, Dec 2014, pp. 2286-2291. 
20. A. Anastasiou, C. Tsirmpas, A. Rompas, K. Giokas, and D. Koutsouris, "3d printing: Basic concepts mathematics and technologies, " in Bioinformatics and Bioengineering (BIBE), 2013 IEEE 13th International Conference on, Nov 2013, pp. 1-4.

21. S. Schulz, I. Ltkebohle, and S. Wachsmuth, "An affordable, 3dprintable camera eye with two active degrees of freedom for an anthropomorphic robot," in Intelligent Robots and Systems (IROS), 2012 IEEE/RSJ International Conference on, Oct 2012, pp. $764-771$.

22. S. Davis, N. Tsagarakis, and D. Caldwell, "The initial design and manufacturing process of a low cost hand for the robot icub," in Humanoid Robots, 2008. Humanoids 2008. 8th IEEE-RAS International Conference on, Dec 2008, pp. 40-45.

23. MAGADUM, RANI A., and DR UTTAM L. BOMBALE. "INTELLIGENT COLOR BASED OBJECT SORTING USING ROBOTICS." International Journal of Robotics Research and Development (IJRRD) 9.1 (2019):43-48

24. SARAVANAKUMAR, S., and MK BADRI NARAYANAN. "THE SERVICE AUTOMATION AND ROBOTICS IN HOSPITALITY INDUSTRY: A STUDY ON BUSINESS IMPLICATIONS." International Journal of Mechanical and Production Engineering Research and Development (IJMPERD) 8.6 (2018):91-100

25. HIPPOLITUS, A. JOSIN, and AYUSH OBEROI. "DESIGN AND DEVELOPMENT OF AN IOT BASED MULTI TERRAIN HUMANOID ROBOT FOOT." International Journal of Mechanical and Production Engineering Research and Development (IJMPERD) 8.6 (2018):143-150

26. Zabbar, Md Ajijul Bin, and Chisty Nafiz Ahmed. "Design \& Implementation of an Unmanned Ground Vehicle (UGV) Surveillance Robot." International Journal of Electrical and Electronics Engineering (IJEEE) 5.6 (2016): 11-20.

27. Gandhi, K. R. U. T. A. R. T. H., et al. "Motion controlled robotic arm." International Journal of Electronics and Communication Engineering (IJECE) 2.5 (2013): 81-86.

28. Bryndin, Evgeniy. "Development of sensitivity and active behavior of cognitive robot by means artificial intelligence." International Journal of Robotics Research and Development (IJRRD) 10.1 (2020): 1-12.

29. NIKITIN, VLADIMIRS, et al. "MULTI-AGENT REMOTE CONTROL SYSTEM FOR EXTINGUISHING FOREST FIRES WITH HETEROGENEOUS ELEMENTS OF A ROBOTIC COMPLEX." International Journal of Mechanical and Production Engineering Research and Development (IJMPERD) 9.6 (2019):25-32

30. NIKHIL, GAJAVALLI VENKATA PAVAN GOPI, and GANDHAM YEDUKONDALU. "ROBOTIC EXOSKELETONS: A REVIEW ON DEVELOPMENT." International Journal of Mechanical and Production Engineering Research and Development (IJMPERD) 9.4 (2019):529-542 